\title{
A review on angle resolved photoemission spectroscopy studies of Fe-based superconductors
}

\author{
J.J. Seo ${ }^{\mathrm{a}}$ and C. Kim* ${ }^{*}$ a \\ ${ }^{\mathrm{a}}$ Department of Physics, Yonsei University, Seoul, Korea
}

(Received 19 June 2014; revised or reviewed 26 June 2014; accepted 27 June 2014)

\begin{abstract}
Since the discovery of iron-based superconductors in 2008, extensive and intensive studies have been performed to find the microscopic theory for the high temperature superconductivity in the materials. Electronic structure is the basic and essential information that is needed for the microscopic theory. Experimentally, angle resolved photoelectron spectroscopy (ARPES) is the most direct tool to obtain the electronic structure information, and therefore has played a vital role in the research. In this review, we review what has been done so far and what is needed to be done in ARPES studies of iron-based superconductors in search of the microscopic theory. This review covers issues on the band structure, orbital order/fluctuation, and gap structure/symmetries as well as some of the theories.
\end{abstract}

Keywords: iron-based superconductors, electronic structures, angle resolved photoemission, microscopic mechanism

\section{INTRODUCTION}

In 2008, Hosono group discovered an iron-based superconductor (IBSC) with a relatively high critical temperature $\left(T_{c}\right)$ of $26 \mathrm{~K}$. It was followed by synthesis of barrage of IBSC compounds, among which was $\mathrm{SmFeAsO}$ with the highest $\mathrm{T}_{\mathrm{c}}$ of $55 \mathrm{~K}$. IBSCs immediately drew much attention because, other than cuprates, they are the only group of transition metal compounds with high $\mathrm{T}_{\mathrm{c}}$. Naturally, the microscopic mechanism behind the high temperature superconductivity in IBSCs was a central issue. In particular, the fact that IBSCs share a similar phase diagram to that of cuprate superconductors raised some hope that study of IBSCs may lead to obtaining the microscopic theory for cuprate superconductivity. For these reasons, there have been extensive and intensive studies on IBSCs.

Finding microscopic mechanism requires thorough understanding of momentum resolved electronic structures because they bare important information that is necessary to build the microscopic theory. Angle resolved photoemission spectroscopy (ARPES) is the most powerful and direct tool to investigate the momentum resolved electronic structure. For that reason, from the very beginning of the iron-pnictide era, ARPES has been intensively used to investigate the electronic structures of iron-based superconductors. For example, the gap symmetry which provides critical information on the pair mediating Bosons can be measured by ARPES [1].

In fact, much information has been obtained on the gap symmetries of various IBSCs by using ARPES. In early days, most of the materials showed full gaps. In combination with the fact that Fermi surface pockets at the

\footnotetext{
* Corresponding author: changyoung@yonsei.ac.kr
}

Brillouin zone (BZ) center and corner have fairly good nesting conditions, the observed full gap appears to suggest that the two gaps have opposite phases with an $S_{ \pm}$ symmetry [2]. However, some theories and experiments on other materials raised possibilities of $s_{++}, d$, and $s+i d$ gap symmetries [3-12]. As a result, there are on-going debates on what the universal mechanism of the iron-based superconductivity is.

ARPES studies have also been performed to investigate properties other than the gap symmetry. IBSCs have multiple phases such as tetragonal and orthorhombic phases in crystalline structure, and antiferromagnetic, nematic, and pseudo gap phases in the electronic aspect. These phases are strongly correlated with the superconducting phase as suggested by some of the theories $[2,13$, 27-31], and thus understanding the mechanism behind these phases may provide us important clues to the microscopic theory of the superconductivity.

Orbital degree of freedom is another important subject. Early ARPES studies showed that IBSCs are multi-orbital systems [14] and that orbital degree of freedom is an important variable in the formation of the superconductivity [15-20]. In regards to the orbital degree of freedom, orbital ordering has been shown to exist [18-20], which raised possibility of orbital fluctuation as the mediating glue.

As described above, intensive and extensive ARPES experiments have been performed and the results are a crucial part of the information needed to build the microscopic theory. In this review, we wish to describe efforts to understand the electronic structures of IBSCs relevant to superconductivity by reviewing important ARPES results. Before we go into superconductivity, we describe structures and phase diagrams of IBSCs in section 2. In 
section 3, we introduce the multi-orbital nature of the electronic structure to discuss the generally observed nesting condition between zone center and corner pockets. Section 4 is devoted to discussion of various superconductivity scenarios of IBSCs while section 5 is on the relationship between the superconductivity and various parameters such as nesting, orbital degree of freedom. In section 6, we will go over gap symmetries from various scenarios and discuss the feasibilities of the scenarios. Finally, we will provide brief discussion on some of the unresolved issues and what needs to be done by using ARPES in section 7.

\section{STRUCTURE AND PHASE DIAGRAM}

The most basic information one needs to know is the crystal structure. As shown in figure 1, various types of IBSC compounds have been discovered [21]. All of the compounds have FeAs or FeSe layers, and it is believed that $\mathrm{Fe} 3 d$ orbitals in these $\mathrm{Fe}$ containing layers are responsible for the superconductivity, magnetism and the phase transitions.

Even though there is some similarity between FeAs layers in IBSCs and $\mathrm{CuO}$ layers in cuprates in many aspects, a notable difference is that As in FeAs layer is located above and below $\mathrm{Fe}$ atoms. As a result, IBSCs have more 3 dimensional (3D) crystal and electronic structures. The As height from the Fe-plane turned out to be an important parameter that greatly affects various physical properties. One of the reasons that each system displays different physical properties is that the As height varies among various systems and so does the resulting electronic structure. It is not only FeAs layers but also atoms/layers below and above FeAs planes that affect the structure and doping of the FeAs layers, resulting in characteristic properties of each system. For this reason, the superconductivity in IBSCs requires understanding the properties of FeAs layers, especially the electronic structure for various As heights and interlayer effects [22-25].

Phase diagram of IBSCs bares much information on the

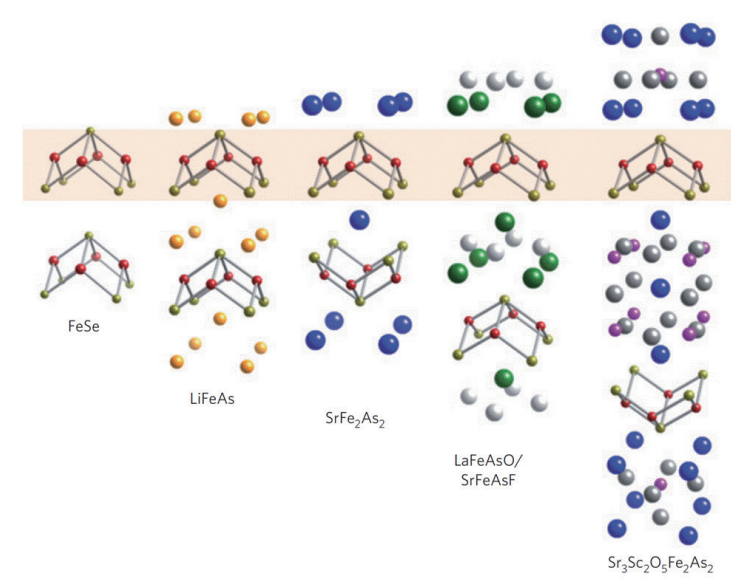

Fig. 1. Crystal structures of various iron-based superconductor compounds. All the compounds contain Fe-As or Fe-Se planes (shaded) [21].
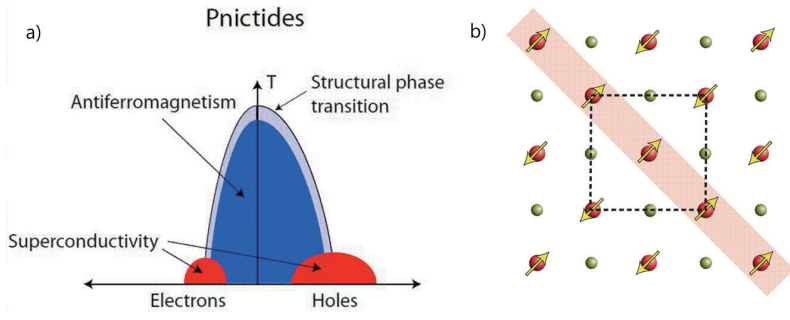

Fig. 2. Typical (a) phase diagram of IBSCs [26] and (b) spin configuration in FeAs layers [21].

superconductivity mechanism. Panel (a) in figure 2 is a schematic of IBSC phase diagram. Parent IBSCs have tetragonal structure and is metallic at room temperature. When the temperature is lowered, it goes through tetragonal to orthorhombic phase transition and obtains antiferromagnetism. For the magnetism, as shown in figure $2 \mathrm{~b}$, the magnetic moments of $\mathrm{Fe}$ atoms align ferromagnetically (antiferromagnetically) along the shorter (longer) crystal axis in the orthorhombic phase. As we dope electron and hole or even apply pressure, the magnetism in the parent compounds begins to be suppressed and the superconducting phase appears at the edge of the antiferromagnetic region. The fact that superconductivity appears at the edge of the superconducting region in the phase diagram for both cuprates and iron-pnictides suggests that the superconductivity has close relationship with magnetism. Numerous theoretical and experimental studies supporting such view have been published.

However, various recent experimental results showed orbital ordering phase with imbalance in $d_{x z}$ and $d_{y z}$ orbital occupations [18-20]. These results suggest a possibility that orbital fluctuation could induce structural and magnetic transitions and could be the superconductivity mediating boson. In fact, theoretical proposals for such possibility have been made [27-30]. For this reason, candidates for the pairing mediating boson in IBSCs include spin and orbital fluctuations, and which one is responsible is still under debate. We will discuss this subject in more detail in section 4.

It was also recently reported that, as shown in figure 3, nematic phase exists in $\mathrm{BaFe}_{2}(\mathrm{As}, \mathrm{P})_{2}$ as observed by magnetic torque measurement [31]. Nematic phase refers to broken 4 fold symmetry in the electronic structure, independent of the crystal structure symmetry. According

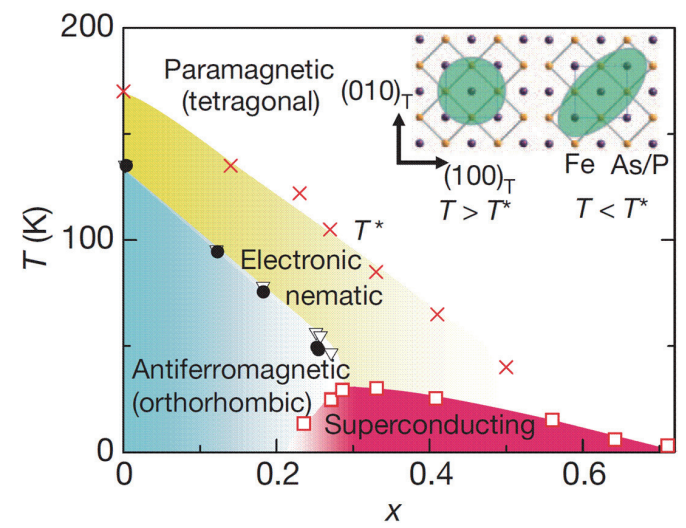

Fig. 3. Phase diagram of $\mathrm{BaFe}_{2}(\mathrm{As}, \mathrm{P})_{2}$ obtained through magnetic torque measurement [31]. 
to the report, nematic phase sets in at a much higher temperature than the structural transition temperature and is suppressed upon doping. It disappears together with superconductivity when doping is too high. These results suggest a close relationship between nematic phase and superconductivity, and it is believed that the order parameter for the nematic phase can be spin fluctuation, orbital fluctuation or coupled fluctuation of the two [14, 32, 33]. Therefore, finding the order parameter for the nematic phase is expected to provide an important clue to the superconductivity in IBSCs.

\section{BAND STRUCTURE}

In section 2, we have discussed various aspects of IBSCs by discussing the crystal structures and phase diagrams. In this section, we will discuss the common features of the IBSC electronic structures obtained by ARPES experiments, in a effort to understand the universal aspect of the superconductivity in IBSCs.

\subsection{Fermi surfaces : nesting condition}

Figure 4 shows Fermi surfaces of various parent compounds obtained by ARPES experiments [34-36]. Common features of the Fermi surface topology is that there are hole pockets at the BZ center and electron pockets at the BZ corner. The number of pockets and their orbital characters differ among different compounds. The reason for such variety in the Fermi surface shape is due to their sensitivity to the As height and interlayer as mentioned in section 2 .

The key aspect of the Fermi surface topology of IBSCs is that there is a good nesting condition between hole and electron pockets. Such a good nesting condition is believed
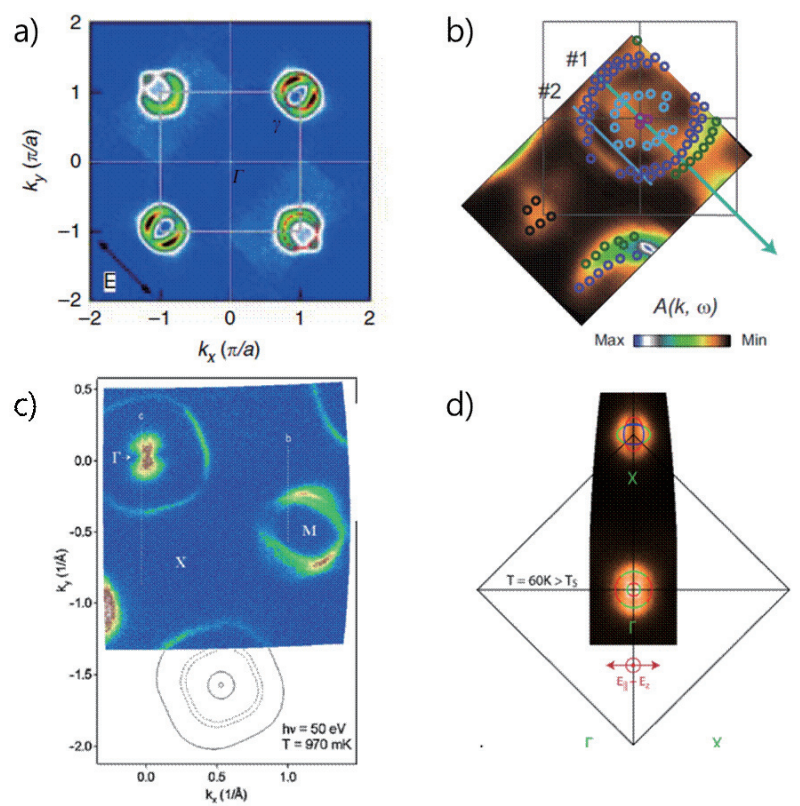

Fig. 4. Experimentally obtained Fermi surface maps of (a) FeSe monolayer [34] (b) La1111 [35], (c) Li111 [36], and (d) NaFeAs [19]. to be important but it is not clearly explained how exactly it contributes to the superconductivity. We will describe in more detail how nesting affects the superconductivity in section 4 which deals with superconductivity scenarios.

\subsection{Multi-orbital nature of the band}

Another important character of the electronic structure is that the bands at the Fermi level originate from

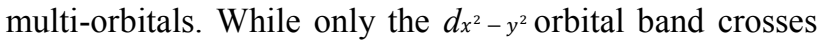
the Fermi level in cuprate superconductors, all five of Fe $3 d$-orbitals contribute to the density of states at the Fermi level in IBSCs. Even though all of the Fe $3 d$-orbitals contribute to near $\mathrm{E}_{\mathrm{F}}$ states in IBSCs, they split into $e_{g}$ and $t_{2 g}$ orbitals if the crystal field is considered, and the main contribution comes from the three $t_{2 g}$ orbitals, $d_{x y}, d_{y z}, d_{x z}$. As a result, these three orbitals are mostly responsible for the superconductivity and other physical properties.

The fact that multi-orbitals contribute to the superconductivity greatly affects the character of the superconductivity in IBSCs. Especially, it raises the possibility of orbital ordering and orbital fluctuation mediated superconductivity discussed in section 2 [27-30]. Its effect in the superconductivity will be discussed in detail in section 4 on superconductivity scenarios. Multi orbital nature of the IBSC bands can be examined through polarization dependent ARPES. Parity selection rule produces polarization dependent ARPES intensities, which allows us to investigate the orbital characters [37]. For more details on the calculation of the ARPES matrix, readers are advised to refer to ref. 37 .

Figure 5 shows the orbital characters of $\mathrm{NaFeAs}$ by using the method described above [19]. The outermost hole band ( $\gamma$ band, blue line) mostly has the $d_{x y}$ character but it obtains significant $d_{y z}$ character near the Fermi energy due to hybridization with second hole band ( $\beta$ band, green line). The inner most band ( $\alpha$ band, red line) has pure $d_{x z}$ character. As for the electron bands at the BZ corner, the inner and outer bands have $d_{x y}$ and $d_{y z}$ characters, respectively. A closer look at figure 4 shows that there is a good nesting condition between the hole and electron pockets as they have similar sizes. However, the intra-orbital nesting condition is found to be relatively poor.

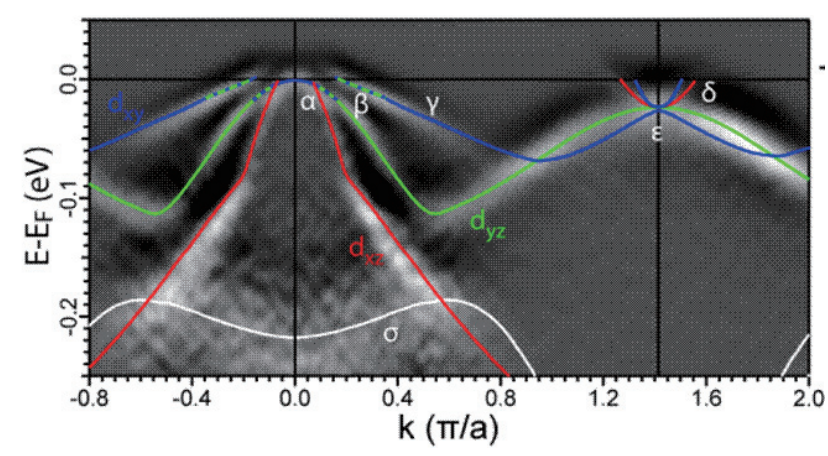

Fig. 5. ARPES data from NaFeAs. Drawn on top of the data are color coded orbital characters of the bands [19]. 


\section{SUPERCONDUCTIVITY SCENARIOS}

In the previous section, we briefly introduced the fact that two most basic characters of the electronic structures, Fermi surface nesting and multi-orbital nature of the bands, are important parameters of superconducting mechanism in IBSCs. In this section, we discuss how these parameters work in each superconductivity scenario.

\subsection{Weak coupling approach}

There are two approaches to understanding superconductivity in IBSCs, weak [2, 38-41] and strong [42-46] couplings. Initially, it was believed that weak coupling approach could explain the superconductivity as electron correlation was thought to be negligible in the metallic phase. However, subsequent experiments showed that the electron correlation was not small, contrary to the initial belief [43]. As a result, there have been continuing efforts to explain the superconductivity with the strong coupling approach. Which of the two is more appropriate is still under debate.

Weak coupling approach includes spin density wave (SDW) fluctuation and orbital fluctuation mediated superconductivity. These scenarios require itinerant electrons and thus the Fermi surface topology plays an important role. In the SDW fluctuation mediated superconductivity scenario, the SDW fluctuation created by the nesting of Fermi surfaces at the BZ center and corner mediates the Cooper pairs as shown in figure 6(a). Plotted in figure 6(b) is the dynamic spin susceptibility. It shows an enhanced but rather broad shape near $\boldsymbol{q}=(\pi, \pi)$ due to the fact that the Fermi surface pocket at the M point is not circular but oval.

In spite of the broadening, within the SDW scenario, spin fluctuation is the strongest magnetic fluctuation and affects the superconductivity in IBSCs. Calculations show that the spin susceptibility diverges when the nesting is due to intra-orbital scattering if multiple-orbital character is considered. This claim is consistent with the experimental observation that the superconducting gap of the $\mathrm{BZ}$ center pocket is almost zero when the $\mathrm{BZ}$ corner $d_{x y}$ electron bands disappear in $\mathrm{KFe}_{2} \mathrm{As}_{2}$ system [77].

In the orbital fluctuation mediated superconductivity scenario, orbital fluctuation occurs due to the orbital exchange process from the additional Coulomb potential that Fe $d$-electrons feel additional when Fe atoms oscillate. Presently, there are two scenarios for orbital fluctuation mediated superconductivity, one with $\boldsymbol{q}=(0,0,0)$ ferro

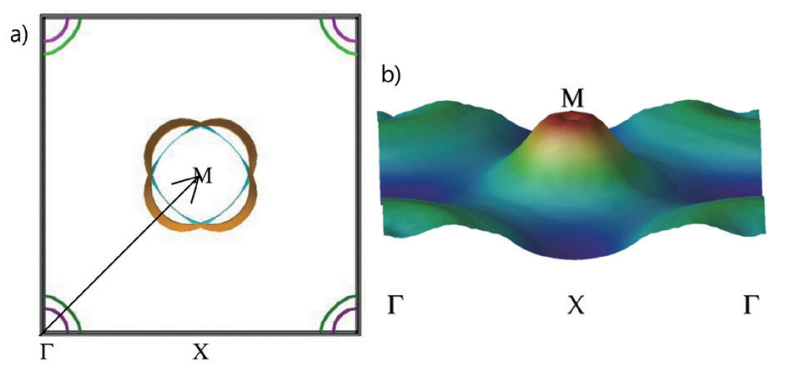

Fig. 6. (a) Nesting condition in IBSC and (b) The spin susceptibility at the M-point due to the nesting [2].
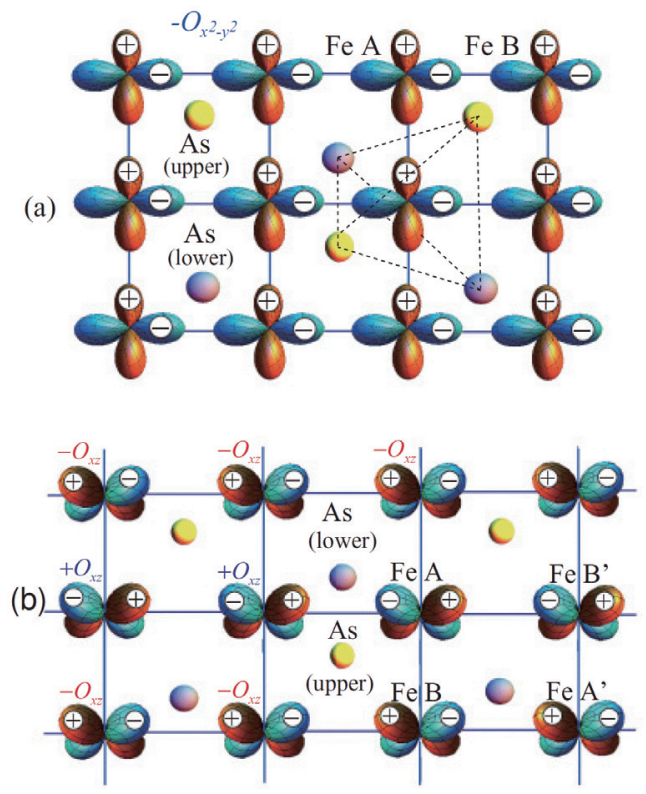

Fig. 7. (a) Ferro orbital and (b) antiferro orbital fluctuation [27]

orbital fluctuation [30] and $\boldsymbol{q}=(\pi, \pi, 0)$ antiferro orbital fluctuation [27-29]. Ferro orbital fluctuation refers to the case where the quadrupole operators $O_{x^{2}-y^{2}}$ at each Fe site are aligned parallel to each other ('ferro'-order) as shown in figure 7(a). In that case, the occupations of $\mid x z>$ and $|y z\rangle$ $d$-orbital states ferro-fluctuate as shown in figure 8(a).

On the other hand, in the antiferro orbital scenario, $O_{x z}$ quadrupole operators are aligned in a strip-type as seen in figure 7(b) and occupations of $|x y>-| y z\rangle$ and $|x y\rangle+|y z\rangle$ states of $d$-orbital antiferro fluctuate as shown in figure 8 (b). In this case, contrary to the spin fluctuation case, the quadrupole susceptibility diverges and the gap size becomes independent of the band. This is supported by the experiments on $(\mathrm{Ba}, \mathrm{K}) \mathrm{Fe}_{2} \mathrm{As}_{2}[59]$.

\subsection{Strong coupling approach}

The strong coupling approach is a way to understand the superconductivity in the large $U$ limit [42-50]. When $U$ is very large, the magnetism in IBSC can be explained in terms of As bridged super-exchange. As shown in figure 9, calculations show that the magnitude of the next nearest neighbor exchange $J_{2}$ is similar to that of the nearest exchange $\mathrm{J}_{1}$ due to the strong As-As hopping, which in turn results in antiferromagnetic strip structure along the $\boldsymbol{q}=(\pi, 0)$ direction.
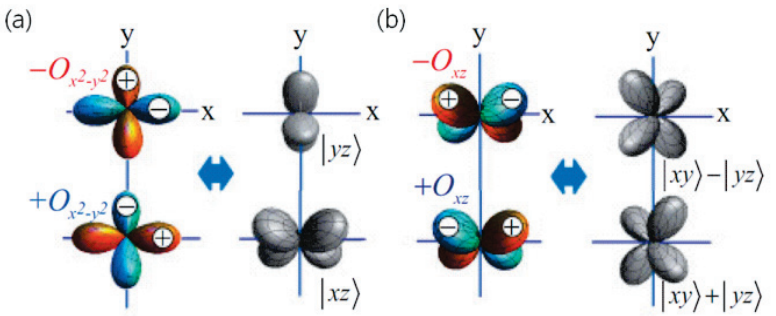

Fig. 8. (a) Ferro orbital fluctuation due to the quadrupole operator $O_{x^{2}-y^{2}}$ and (b) antiferro orbital fluctuation from $O_{x y}[27]$. 

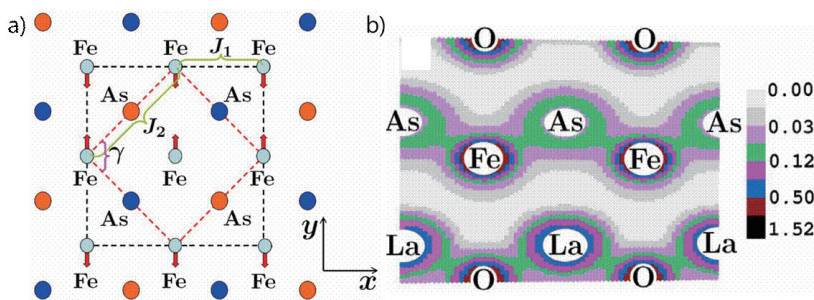

Fig. 9. (a) Schematic top view of the FeAs layer and (b) charge density distribution in the (110) plane [45].

In the strong coupling approach, pairing is mediated by the fluctuation of the local antiferromagnetism as is the case for cuprate superconductivity. Therefore, the superconductivity is not determined by the Fermi surface topology but by the shape of the BZ unlike in the weak coupling approach. Experimental results that support the local antiferromagnetic fluctuation scenario include superconductivity without the nesting condition between Fermi surface pockets at BZ center and corner, and the high magnetic moment values that cannot be explained within the SDW picture [50-55]. In the picture, the doping dependent phase diagram can be understood as being due to changes in the super-exchange interaction for different dopants. This is similar to the cuprate superconductivity in which superconductivity appears when antiferromagnetis$\mathrm{m}$ is suppressed as the doping increases.

However, it does not appear that one can explain the superconductivity based purely on a single approach. Both local and itinerant characters of the electrons are observed for IBSC materials. Various experiments show that there is orbital dependent correlations strength. As a result, it is somewhat accepted in the community that the superconductivity has a dual nature $[56,57]$. Therefore, it is imperative to investigate origin of the dual nature of the electrons in the IBSC materials and how these traits results in various phases.

\section{SUPERCONDUCTIVITY AND BAND STRUCTURE}

In section 5, we discuss the possible relationship among nesting, orbital ordering and superconductivity by looking at the band structure in detail

\subsection{Nesting condition}

We have stated in section 4 that the nesting condition is an important parameter for the SDW spin fluctuation and antiferro orbital fluctuation scenarios in the weak coupling approach. In 5.1, we will try to understand the relationship between nesting and superconductivity from how the nesting condition changes as the superconductivity sets in.

Figure10 shows Fermi surface maps of $\mathrm{BaFe}_{2} \mathrm{As}_{2}$ with various dopants. The Fermi surface for the parent material in figure 10(a) shows a good nesting condition between pockets at the BZ center to corner. The strong nesting condition readily explains the existence of the static magnetic order rather than magnetic instability in the parent compound.

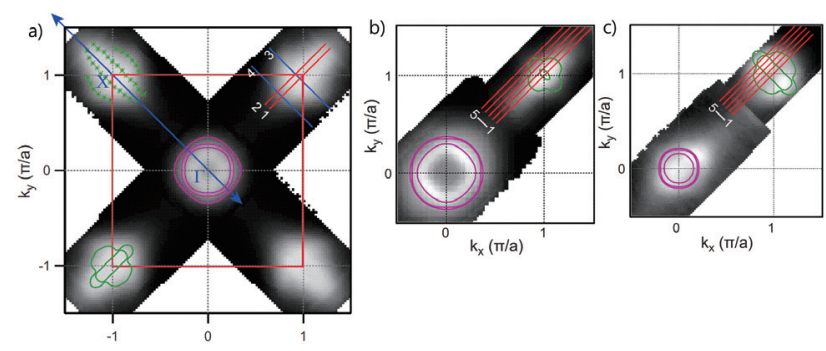

Fig. 10. Fermi surfaces and nesting conditions in (a) parent $\mathrm{BaFe}_{2} \mathrm{As}_{2}$, (b) $\mathrm{K}$ doped $\mathrm{BaFe}_{2} \mathrm{As}_{2}$, and (c) Co-doped $\mathrm{BaFe}_{2} \mathrm{As}_{2}$ [58].

Plotted in figure 10 (b) is the Fermi surface map of $(\mathrm{K}, \mathrm{Ba}) \mathrm{Fe}_{2} \mathrm{As}_{2}$. It shows that the hole pocket size increased while the electron pocket shank, which means that K dopes holes into the system. As the doping increases, the nesting condition becomes poorer and at the same time superconductivity appears. On the other hand, Co-doped case in figure $10(\mathrm{c})$ shows that the trend is opposite to that of the K-doping case, that is, the hole pocket becomes smaller and electron pocket get larger as the Co-doping increases. As was the case in $(\mathrm{K}, \mathrm{Ba}) \mathrm{Fe}_{2} \mathrm{As}_{2}$, superconductivity sets in as the nesting becomes weaker. These observations of anti-correlation between the superconductivity and nesting condition supports the weak coupling approach.

According to the SDW fluctuation scenario, the magnetic susceptibility due to the spin fluctuation becomes divergent when the nesting conditions are satisfied. Therefore, $T_{c}$ is higher when the intra-orbital nesting condition is strong. In the following, we would like to show experimental evidences for the close relationship between the intra-orbital nesting and superconductivity.

Figure 11 plots the Fermi surface maps of $\mathrm{Ba}_{1-\mathrm{x}} \mathrm{K}_{\mathrm{x}} \mathrm{Fe}_{2} \mathrm{As}_{2}$ in the over doped region, $\mathrm{x}=0.5$ and 0.6 [59]. One can see that the inner electron pocket disappears as the doping $x$ increases. Band calculation results shows that the outer hole and inner electron pockets $d_{x^{2}-y^{2}}$ and $d_{x y}$ orbital characters, respectively. In addition, the intra-orbital scattering decreases as the doping increases as the inner pocket disappears.

How that affects the superconducting gap size is plotted in figure 12. The gap size of the inner band linearly ecreases as the doping increases in the over doped region
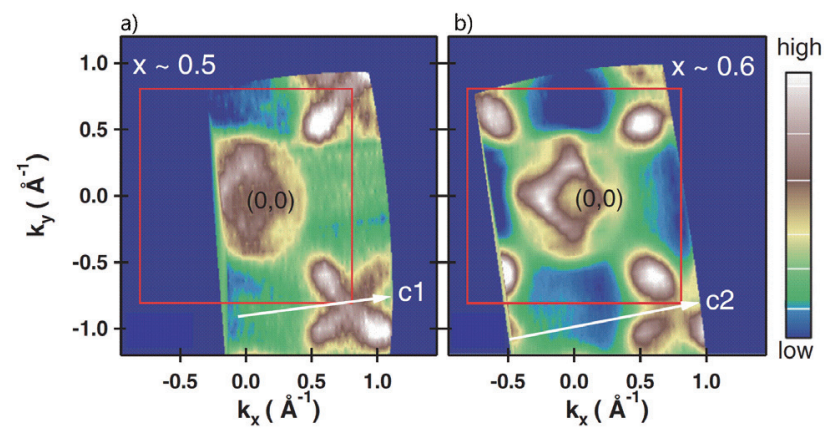

Fig. 11. Fermi surfaces of $\mathrm{Ba}_{1-x} \mathrm{~K}_{x} \mathrm{Fe}_{2} \mathrm{As}_{2}$ for (a) $x=0.5$ and (b) $x=0.6[59]$. 


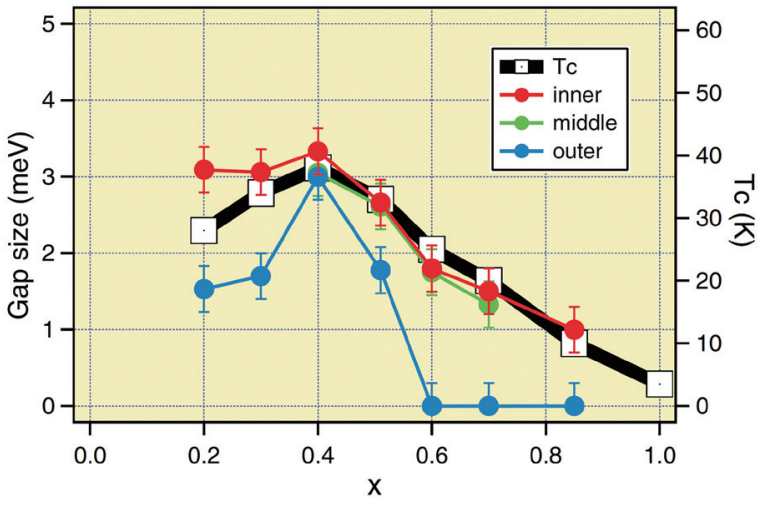

Fig. 12. Orbital dependent superconducting gap as a function of $x$ in $\mathrm{Ba}_{1-x} \mathrm{~K}_{x} \mathrm{Fe}_{2} \mathrm{As}_{2}$ [59].

until the complete substitution of $\mathrm{Ba}$ by $\mathrm{K}, \mathrm{KFe}_{2} \mathrm{As}_{2}$, while the gap of the outer band abruptly drops to zero at $x=0.6$ at which the inter-orbital nesting disappears. This experimental result is an important supporting evidence for the role of inter-orbital nesting in the formation of superconductivity.

The data for $\mathrm{Ba}_{1-x} \mathrm{~K}_{x} \mathrm{Fe}_{2} \mathrm{As}_{2}$ in figure 12 is a supporting evidence not only for the SDW fluctuation scenario but also for the orbital fluctuation scenario. According to the orbital fluctuation scenario, the susceptibility for the orbital fluctuation becomes divergent when inter-orbital nesting is met. If the superconductivity is induced by the inter-orbital nesting, the superconducting gap should be the same independent of the orbital. In figure 12, we see that the three bands have the same gap size for the optimal doping $x=0.4$. This observation implies a possibility for orbital fluctuation mediated superconductivity. In addition, the results in figures 11 and 12 suggest that the microscopic mechanism for IBSCs can vary depending on the doping.

It was recently reported that only a certain orbital is important in the formation of superconductivity. Figure 13 depicts the $\mathrm{k}_{\mathrm{z}}$ dependent ARPES intensity at the Fermi energy from $\mathrm{Ba}\left(\mathrm{Fe}_{1-x} \mathrm{Ru}_{x}\right)_{2} \mathrm{As}_{2}$ for various $x$ values [60]. Figures (a) and (g) show a good nesting condition between the hole and electron bands. However, in the optimal doping data in figures (b) and (h), we see that the inner hole pocket gets a significant $\mathrm{k}_{\mathrm{z}}$ dispersion and as a results the nesting condition becomes fairly weak. In the over doping region shown in figures (c) and (i), the Fermi surface becomes even more 3 dimensional and the nesting condition almost completely vanishes. The observed change in the Fermi surface topology into more 3 dimensional one supports the spin fluctuation scenario. On the other hand, the $\beta\left(d_{x y}\right)$ band keeps the nesting condition even in the over doping region but it hardly affects the $T_{c}$. This fact implies that the nesting condition for the $\alpha$, and $\alpha$ ' $\left(d_{x z}\right.$ and $\left.d_{y z}\right)$ bands are more important parameters.

So far, we have pointed out that superconductivity in IBSC is enhanced in the weak coupling approach as nesting becomes weaker. Contrary to this view, however, there is a recent report that superconductivity is enhanced when the nesting condition for a specific orbital is strong [45]. As shown in figure $14, \mathrm{NaFe}_{1-x} \mathrm{Co}_{x}$ As has the highest $\mathrm{T}_{\mathrm{c}}$ when $d_{x z}$ and $d_{y z}$ orbitals have nearly perfect nesting.

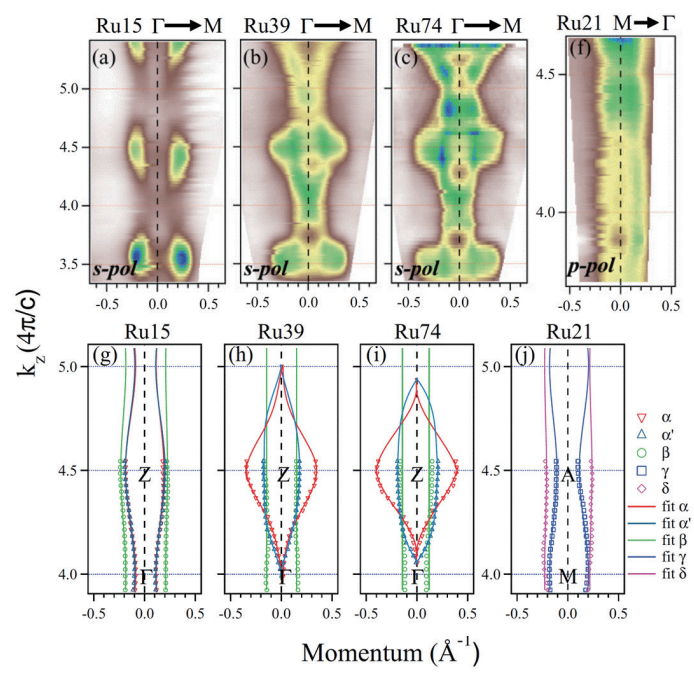

Fig. 13. $\mathrm{k}_{\mathrm{z}}$ dispersion and nesting condition as a function of doping $x$ in $\mathrm{BaFe}_{2-x} \mathrm{Ru}_{x} \mathrm{As}_{2}$. ARPES intensity at the Fermi level near the $\Gamma$-point as a function of $k_{z}$ for (a) under doping, (b) optimal doping, and (c) over doping. (g)-(i) are extracted dispersions from panels (a)-(c), respectively. (f) and (j) are similar plots for $21 \% \mathrm{Ru}$ doping for the electron band near M-point [60].
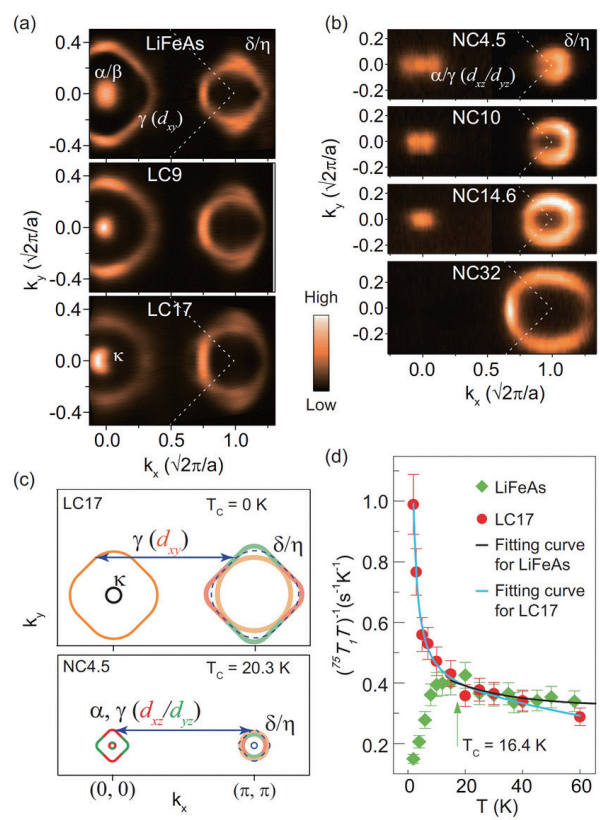

Fig. 14. Fermi surface topology change upon Co-doping for (a) $\mathrm{LiFeAs}$ and (b) NaFeAs. (c) Nesting conditions for $\mathrm{LiFe}_{0.83} \mathrm{Co}_{0.17} \mathrm{As}$ and $\mathrm{NaFe}_{0.96} \mathrm{Co}_{0.04} \mathrm{As}$. (d) Spin relaxation time for $\mathrm{LiFeAs}$ and $\mathrm{LiFe}_{0.83} \mathrm{Co}_{0.17} \mathrm{As}$ obtained by $\mathrm{NMR}$ [61].

This fact may support the notion that a good nesting between $d_{x z}$ and $d_{y z}$ enhances the superconductivity.

\subsection{Superconductivity without nesting}

We have so far discussed various experimental results that show strong nesting conditions between some of the orbitals. Those results seem to support the weak coupling approach but various and sometimes seemingly inconsistent results may even make us believe that nesting 

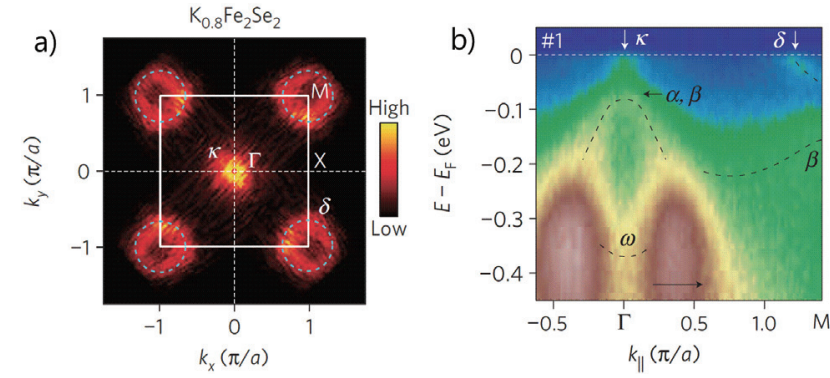

Fig. 15. (a) Fermi surfaces and (b) band dispersions of $\mathrm{K}_{0.8} \mathrm{Fe}_{2} \mathrm{As}_{2}$ [63].

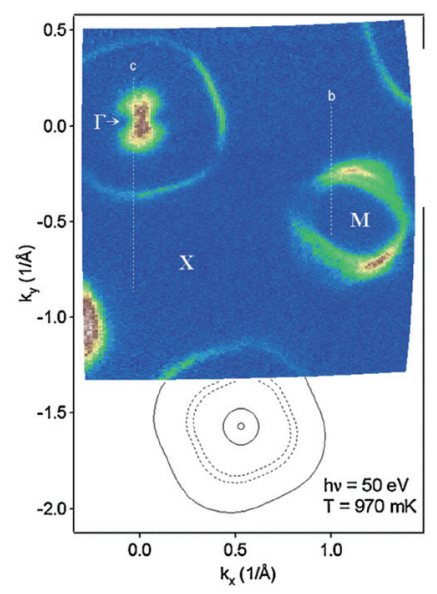

Fig. 16. LiFeAs Fermi surface with poor nesting condition [36].

is not important to the formation of superconductivity. In 5.2, we discuss supporting results for the latter view.

As seen in figure $15, \mathrm{~K}_{0.8} \mathrm{Fe}_{2} \mathrm{As}_{2}$ has a large electron pocket at the M-point but no hole pocket at the $\Gamma$-point [63]. Therefore, so far discussed $(\pi, 0)$ nesting condition cannot be obtained. In spite of the fact, it possesses a $T_{c}$ of $30 \mathrm{~K}$. There are other materials that display superconductivity without or very poor nesting conditions, including $\mathrm{Cs}_{0.8} \mathrm{Fe}_{2} \mathrm{As}_{2}$ [64] and LiFeAs [62].

$\mathrm{LiFeAs}$ is rather peculiar in that the parent compound is superconducting and has no structural and magnetic transitions. This system has three hole pockets at the $\Gamma$-point which have almost no nesting condition with the electron pockets at the M-point as seen in figure 16. Yet it has a $T_{c}$ of $17 \mathrm{~K}$. These materials with high $T_{c}$ but no or poor nesting conditions cast a challenging task for the weak coupling approach. More thorough research has to be performed to see if the nesting is indeed the key ingredient for the superconductivity for IBSCs.

\subsection{Orbital ordering phase}

We now turn our attention to orbital fluctuation scenario and its necessary condition, orbital order phase. Confirmation of an orbital order phase is the first step to prove the existence of orbital fluctuation which may induce structure and magnetic transitions and mediate the superconductivity [18-20]. The orbital order that can be studied by ARPES is the ferro-orbital ordering in which there is an imbalance in the $d_{y z}$ and $d_{x z}$ orbital occupations.
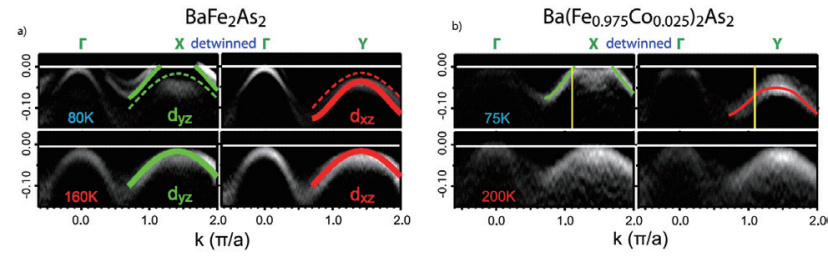

Fig. 17. (a) $d_{x z}, d_{y z}$ bands and orbital order in $\mathrm{BaFe}_{2} \mathrm{As}_{2}$. (b) Orbital order in $\mathrm{Ba}\left(\mathrm{Fe}_{0.975} \mathrm{Co}_{0.025}\right)_{2} \mathrm{As}_{2}$ [18].

Figure 17(a) is the ARPES data from $\mathrm{BaFe}_{2} \mathrm{As}_{2}$ along the $\Gamma-X$ and $\Gamma-Y$ high symmetry cuts for different temperatures. With the help of polarized light, it was found that the states at the X-point are from $d_{y z}$ orbital while those at the Y-point are primarily from $d_{x z}$ orbital. First of all, the data taken at $\mathrm{X}$ - and $\mathrm{Y}$-points above the structural transition temperature $(160 \mathrm{~K})$ show that the two bands are degenerate and that there is a 4-fold symmetry. This means that the two orbitals are equally occupied. If we look at the data taken below the structural transition temperature of 80 $\mathrm{K}$, we find $d_{y z}$ band has shifted up while $d_{x z}$ band has shifted down in energy. Such behavior is also seen $\mathrm{Ba}\left(\mathrm{Fe}_{0.975} \mathrm{Co}_{0.025}\right)_{2} \mathrm{As}_{2}$ in figure 17(b). The fact that the two bands moved in opposite directions means that the $d_{x z}$ orbital is now more occupied than the $d_{y z}$ orbital. That is, there is a ferro-orbital ordering.

Ferro-orbital order is also found in $\mathrm{LiFe}_{1-\mathrm{x}} \mathrm{Co}_{\mathrm{x}} \mathrm{As}$ [67] and $\mathrm{NaFeAs}$ [19]. In the case of LiFeAs, energy split between $d_{x z}$ and $d_{y z}$ orbital bands is observed at the $\Gamma$-point. The split is the largest for the parent material (which has the highest $\mathrm{T}_{\mathrm{c}}$ ), decreases as the doping increases, and eventually becomes degenerate in the over-doping region. This result puts more weight on the orbital fluctuation scenario than SDW picture in a sense that the gap symmetry of LiFeAs cannot be explained by the SDW fluctuation scenario. So far, whether existence of orbital order is universal among IBSCs is not clear yet. Therefore, systematic studies on orbital order for IBSCs are essential in understanding the role of the orbital fluctuations in the superconductivity.

\section{GAP SYMMETRY}

In section 5, we have covered ARPES results that support various scenarios. In a short summary, the relationship between nesting and superconductivity varies over different materials. For example, in some materials, superconductivity exists in spite of lack of nesting condition. We also see that orbital order exists in multiple IBSC systems.

We note that the results we have discussed so far are not directly connected to the microscopic mechanism. We only try to indirectly understand the mechanism through the characteristics in the electronic structure (nesting and energy split, for example). On the other hand, we also have other means to approach the superconducting mechanism more directly in which we look at the symmetry of the Cooper pairs. In this section, we wish to discuss the gap symmetry and try to understand superconducting 


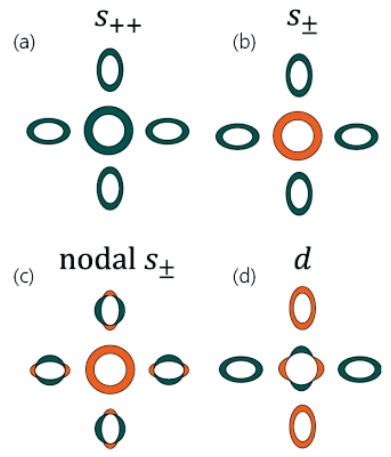

Fig. 18. Schematics of $s_{++}, s_{ \pm}$, nodal $s_{ \pm}$, and $d$-wave gap symmetries [1].

mechanism based on the gap structures measured by ARPES [1].

\subsection{Gap symmetry - theory}

The most direct information on the superconducting mechanism is the gap symmetry. That is because the symmetry of the Cooper pair wave function is directly reflected on the gap symmetry and one can thus retrace the pairing symmetry by studying the gap symmetry. Once the symmetry of the Cooper pair wave function is known, one can pin down the mechanism because pairing symmetry is different for different scenarios.

Figure 18 illustrates gap symmetries expected for different pairing mechanisms in IBSC [1]. $s_{++}$symmetry in figure 18(a) is expected in the case of orbital fluctuation mediated superconductivity. The phases of superconducting gaps on the BZ center and corner pockets are the same, and the gap has 4-fold symmetry. $s_{ \pm}$gap symmetry in $18(\mathrm{~b})$ appears when the superconductivity is mediated by the spin fluctuation. Contrary to the $s_{++}$case, the phases of the $\mathrm{BZ}$ center and corner gaps are opposite. This is due to the fact that electrons on BZ center and corner are paired and spin fluctuation mediated pairing requires a sign change. The node required for the sign change is not seen because there is no Fermi surface between the two pockets. $s_{ \pm}$also possess 4-fold symmetry.

Nodal $s_{ \pm}$in $18($ c) is not much different from the $S_{ \pm}$symmetry, but there is an accidental node. In this case, the accidental node is not from a symmetry reason but from a weak quasi-nesting condition induced by the 3D Fermi surface when the doping increases. On the other hand, $d$-wave symmetry in $18(\mathrm{~d})$ is caused by local antiferro spin fluctuations and 4 nodes exist on the hole pockets. The nodes result from a real symmetry change and the gap has 2-fold symmetry.

While SDW fluctuation scenario necessarily requires $S_{ \pm}$Symmetry, local antiferro spin fluctuation scenario could result in either $s_{ \pm}$or $d$-wave symmetry. However, as shown in figure 19, $d$-wave symmetry has a rather small gap size at the BZ corner, contrary to the $s_{ \pm}$case. The large $\mathrm{T}_{\mathrm{c}}$ 's and gap sizes of IBSCs therefore seem to suggest that $s_{ \pm}$wave is more probable even for the local antiferro spin fluctuation case.

Even though both SDW and local antiferro spin fluctuations could lead to the $s_{ \pm}$gaps, there is some difference between the two cases. Since the shapes of the
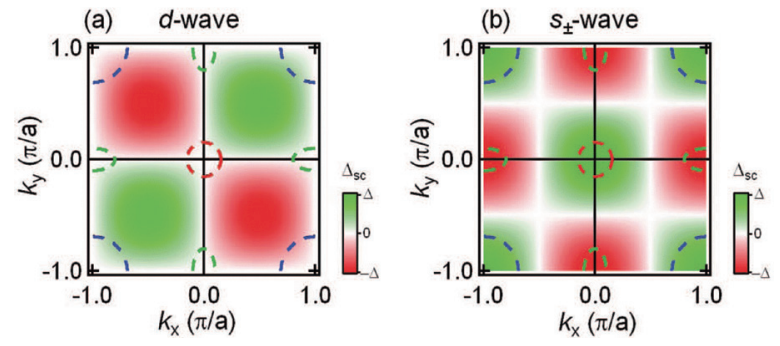

Fig. 19. (a) $d$-wave and (b) $s_{ \pm}$wave gap structure expected in the strong coupling approach [73].

hole and electron pockets are different, the superconducting gap has to be enhanced at the quasi-nested Fermi momentum $\mathrm{k}_{\mathrm{F}}$, resulting in an anisotropic gap structure. On the other hand, the gap should be an isotropic full gap as the gap size depends only on the BZ shape for local antiferro spin fluctuation.

We have discussed various gap symmetries for different microscopic mechanisms. If we can directly determine the gap symmetry through electronic structure studies, we can get close to understanding the microscopic mechanism of IBSC. However, a technique that can directly measure the gap symmetry does not exist yet. ARPES can directly measure the gap size but is insensitive to the phase of the gap. Therefore, it cannot discern $s_{++}$and $s_{ \pm}$gap symmetries. Yet, the anisotropy in the gap and existence of nodes can be determined by ARPES which could provide invaluable clues for the superconducting mechanism.

\subsection{Gap structure - experiments}

Gap anisotropy and nodes obtained through gap size measurements can provide invaluable information on the superconducting mechanism. Isotropic gap can be a supporting evidence for $s_{ \pm}$gap in local antiferro spin fluctuation medicated SC. Meanwhile, anisotropic gap can result from an incomplete nesting in the SDW fluctuation mediated SC scenario $\left(s_{ \pm}\right)$or $s_{++}$symmetry in the orbital order fluctuation scenario. In the following, we would like to survey various ARPES results on gap measurements.

\subsubsection{Isotropic gap structure}
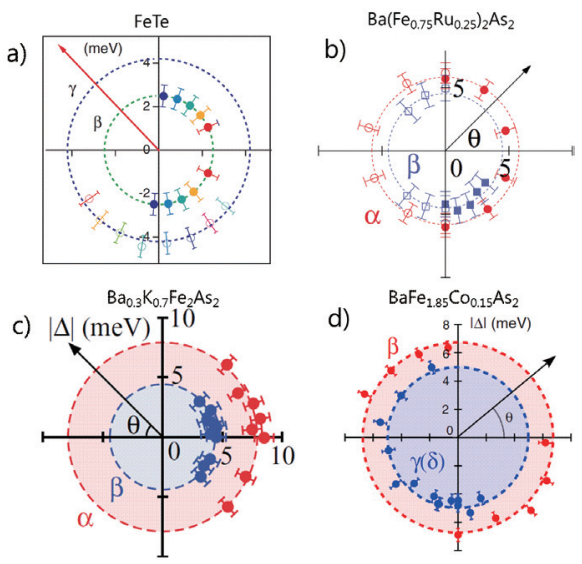

Fig. 20. Band dependent superconducting gaps measured by ARPES as a function of angle for (a) FeTe [69], (b) $\mathrm{Ba}\left(\mathrm{Fe}_{0.75} \mathrm{Ru}_{0.25}\right)_{2} \mathrm{As}_{2}$ [70], (c) $\mathrm{Ba}_{0.3} \mathrm{~K}_{0.7} \mathrm{Fe}_{2} \mathrm{As}_{2}$ [71], and (d) $\mathrm{BaFe}_{1.85} \mathrm{Co}_{0.15} \mathrm{As}_{2}[72]$. 

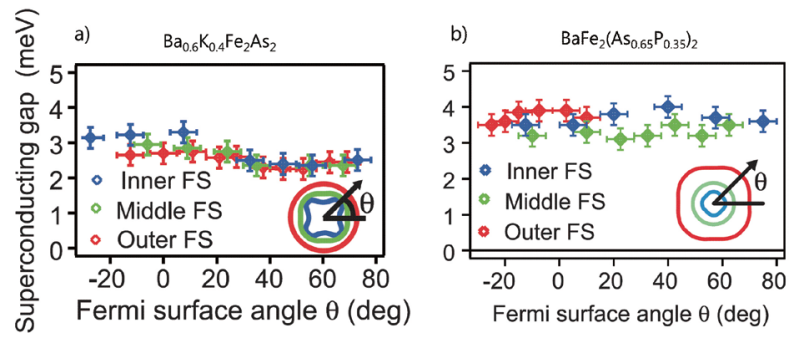

Fig. 21. Band dependent gap size for (a) $\mathrm{Ba}_{0.4} \mathrm{~K}_{0.6} \mathrm{Fe}_{2} \mathrm{As}_{2}$ and (b) $\mathrm{BaFe}_{2}\left(\mathrm{As}_{0.65} \mathrm{P}_{0.35}\right)_{2}[74]$.
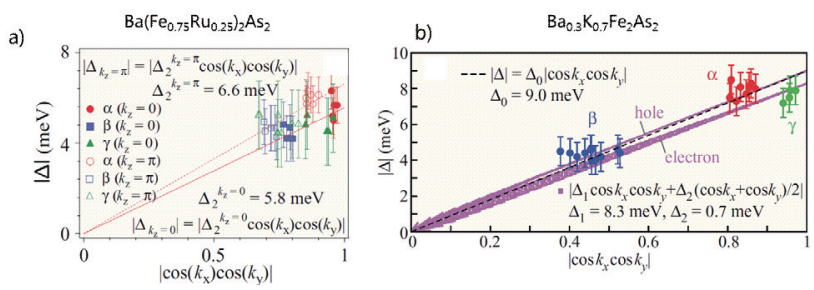

Fig. 22. Cosine fit of the gap for (a) $\mathrm{Ba}\left(\mathrm{Fe}_{0.75} \mathrm{Ru}_{0.25}\right)_{2} \mathrm{As}_{2}$ [70] and (b) $\mathrm{Ba}_{0.3} \mathrm{~K}_{0.7} \mathrm{Fe}_{2} \mathrm{As}_{2}$ [71]

Figure 20 plots the band dependent gap size as a function of angle for various materials. Isotropic but band dependent gap structures have been observed in FeTe [69], $\mathrm{Ba}\left(\mathrm{Fe}_{0.75} \mathrm{Ru}_{0.25}\right){ }_{2} \mathrm{As}_{2} \quad$ [70], $\mathrm{Ba}_{0.3} \mathrm{~K}_{0.7} \mathrm{Fe}_{2} \mathrm{As}_{2} \quad$ [71], and $\mathrm{BaFe}_{1.85} \mathrm{Co}_{0.15} \mathrm{As}_{2}$ [72]. This observation is inconsistent with the SDW scenario in the fact that these materials possess different Fermi surface topologies.

Different from the results in figure 20, gap measurements in figure 21 show isotropic and band independent gap size [74]. Figure 21(a) shows the gap on hole pockets of $\mathrm{Ba}_{0.6} \mathrm{~K}_{0.4} \mathrm{Fe}_{2} \mathrm{As}_{2}$ at the $\mathrm{Z}$-point. Not only the gap size isotropic but also it is the same on all three bands of inner, middle and outer pockets. This is a trait of $s_{++}$gap symmetry of the orbital fluctuation mediated superconductivity.

Figure 22 shows the fitting results of the gap functions by cosine function which is expected in the strong coupling approach [70, 71]. Gaps on all bands for both $\mathrm{Ba}\left(\mathrm{Fe}_{0.75} \mathrm{Ru}_{0.25}\right)_{2} \mathrm{As}_{2}$ and $\mathrm{Ba}_{0.3} \mathrm{~K}_{0.7} \mathrm{Fe}_{2} \mathrm{As}_{2}$ are nicely fitted by the $\cos \mathrm{k}_{\mathrm{x}} \cos \mathrm{k}_{\mathrm{y}}$ function, which once again is an evidence for the strong coupling.

\subsubsection{Anisotropic nodeless gap structure}

Anisotropic gap structure measured by ARPES has been recently reported $[75,76]$. LiFeAs is one of them and shows a clear anisotropic gap structure [75]. Figure 23 is
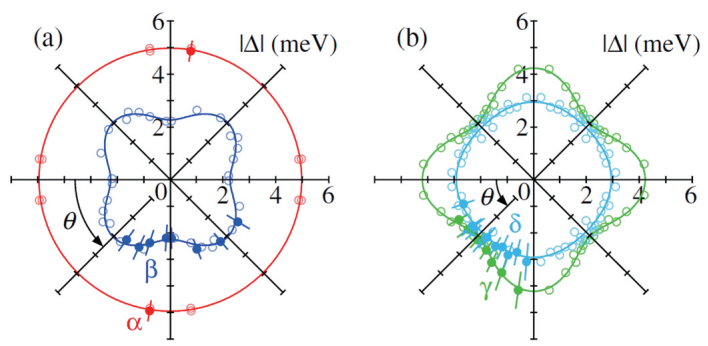

Fig. 23. Band dependent gap sizes of LiFeAs for (a) hole bands and (b) electron bands [75].

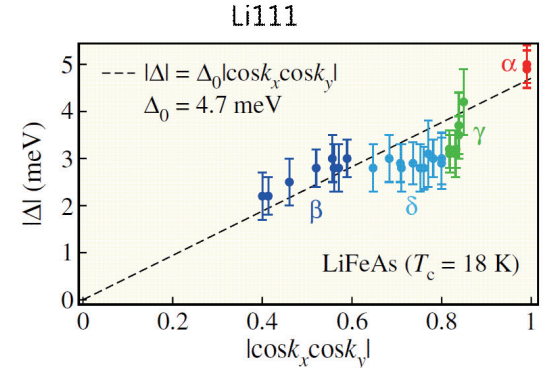

Fig. 24. Fitting results of the LiFeAs gap size within the strong coupling approach [75].

the gap structure of LiFeAs, and shows that the inner pocket at the $\Gamma$-point has an isotropic gap while the outer band has an anisotropic one. The electron pockets show a similar trend; while the outer pocket shows an isotropic gap, the inner pocket has an anisotropic gap.

The fact that the angle for the maximum gap for the hole band is $45^{\circ}$ rotated from that for the inner electron band is not explained within the SDW fluctuation mediated superconductivity scenario. Meanwhile, there was a theoretical report that such structure can be explained within the orbital fluctuation mediate, $s_{++}$symmetry scenario. Gap simulation that takes orbital fluctuation into account can explain the experimental observation that the gap maxima in the $\beta$ hole pocket is $45^{\circ}$ rotated from that for the gap maxima in the $\gamma$ electron pocket.

Anisotropic gap structure can also be explained within the strong coupling approach which is normally supposed to account only for isotropic full gap [73]. It is possible to explain the strong anisotropic gap of LiFeAs if we consider the character of the Fermi surface topology. Unlike other materials, LiFeAs has hole bands with broken rotational symmetry. When this fact is considered, the anisotropy in the hole band can be explained. Figure 24 shows the fitting results of the gap structure within the strong coupling approach. However, it is still not possible to explain the anisotropy in the electron band within the strong coupling approach.

\subsubsection{Nodal gap structure}

Nordal gap structures has been reported for $\mathrm{KFe}_{2} \mathrm{As}_{2}[77]$ and $\mathrm{BaFe}_{2}(\mathrm{As}, \mathrm{P})_{2}[78]$ as shown in Figure 25. In the case of $\mathrm{KFe}_{2} \mathrm{As}_{2}$, high resolution laser ARPES taken at the Z-point shows that inner and middle hole pockets have nodeless anisotropic gap and octet-line nodes, respectively, while the outer band has almost zero gap. The explanation for the existence of nodes in the middle pocket is that the states near the X-point do not have $d_{z^{2}}$ character, which results in a weak intra-orbital scattering. Finally, intra Fermi surface phase sign change becomes easier and thus nodes can be formed. Likewise, almost negligible gap size of the outer band can be explained within intra-orbital, inter-band scattering scenario. Nesting condition dependent gap size change, existence of the node and anisotropic gap structure show that the material has nodal $s_{ \pm}$symmetry

ARPES measurements on $\mathrm{BaFe}_{2}\left(\mathrm{As}_{0.7} \mathrm{P}_{0.3}\right)_{2}$ reveal that all three hole pockets have isotropic gaps while the inner and outer electron pockets have line node and isotropic gap, respectively [78]. The gap structure on the inner electron band plotted in figure 25(b) shows a node in the direction 


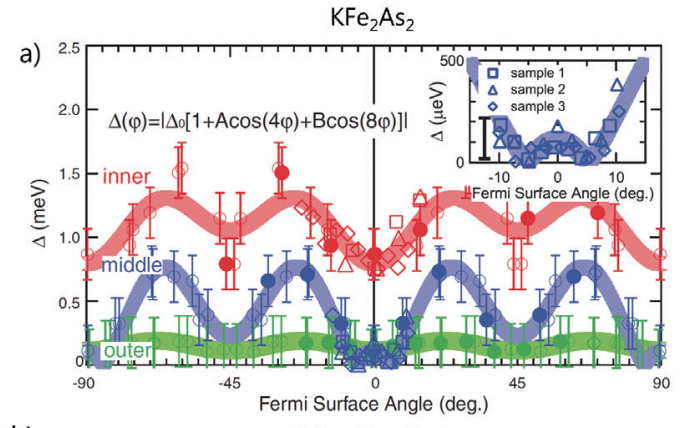

b)

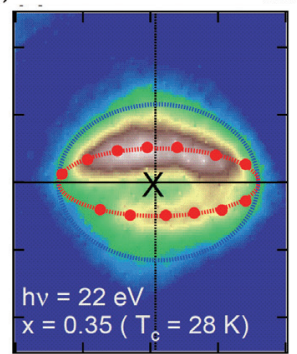
$\mathrm{BaFe}_{2}\left(\mathrm{As}_{0.7} \mathrm{P}_{0.3}\right)_{2}$

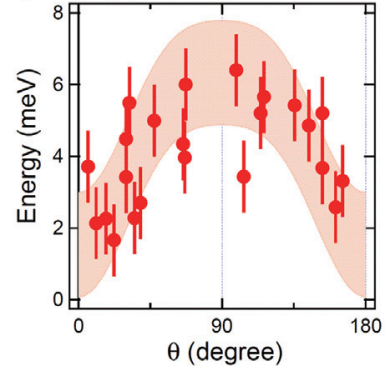

Fig. 25. (a) Band and angle dependent gap size of $\mathrm{KFe}_{2} \mathrm{As}_{2}$ at the Z-point [77]. (b) Fermi surface and angle dependent gap size for $\mathrm{BaFe}_{2}\left(\mathrm{As}_{0.7} \mathrm{P}_{0.3}\right)_{2}[78]$.

toward the $\Gamma$-point. However, it is difficult to call it a nodal $s_{ \pm}$symmetry purely based on the observation of nodes. The outer hole pocket that has $d_{x y}$ orbital near the $\Gamma$-point has very little contribution from the $d_{x y}$ orbital. Then, the intra-orbital scattering with the states at the M-point (consisting of purely $d_{x z}, d_{y z}$, and $d_{x y}$ ) becomes difficult. According to the spin fluctuation mechanism for which intra-orbital and inter-band scatterings are important, hole band must have horizontal node. However, it has not been experimentally observed. In addition, the fact that the gap size remains independent of the bands in spite of the different orbital characters is hard to understand. These questions raise the importance of the inter-orbital scattering and suggest a possibility for spin and orbital fluctuation mediated superconductivity.

We have discussed three types of gap structures. Most of the materials with exceptions of a handful of systems exhibit isotropic gap structures and band dependent gap sizes. This fact implies that the superconductivity can be in general explained within the strong coupling approach but band independent gap size in a few materials and the peculiar gap structure of LiFeAs pose an issue that cannot be resolved within the strong coupling approach.

\section{UNRESOLVED ISSUES AND FURTHER STUDIES}

Extensive studies have been performed on IBSCs and they have produced important experimental results. A variety of materials have been synthesized and physical properties have been studied by using various techniques including transport and photoemission. Recent developments in sample growth have allowed us to have high quality single crystals. This in turn made it possible to have better quality data and allowed us to see what was not seen before. Such progress in experiments will make it possible to answer the questions that have not been answered.

The eventual goal of the research on IBSCs is finding the microscopic mechanism for the superconductivity. However, as we have seen earlier, there are many questions to be answered before we finally achieve the goal. Here list some of the issues in the field have not been resolved.

The first issue that has to be clearly resolved is whether the electrons on IBSCs are itinerant or local. Is nesting essential in the formation of superconductivity and magnetism? There have been numerous experimental reports but consensus has not been made [79-81]. Even though a somewhat compromised view of dual nature is seriously discussed, its understanding is neither systematic nor clear. In this respect, the approach to the microscopic mechanism for superconductivity will be much easier if we understand the nature of the carriers.

The second issue involves structural and magnetic transitions. In IBSCs, it is believed that structural and magnetic transitions are intimately coupled. Therefore, clear understanding of structure and magnetic transitions can provide important clues to the microscopic mechanism for the superconductivity. They have also been investigated extensively but clear understanding of the transitions are lacking. In this regards, there have been recent reports that the transitions are related to nematic phases [82]. Orbital order or spin fluctuation is considered as the order parameter for the nematic phase, but how the transitions are coupled is in debate and needs further studies.

When Fe is substituted by another transitions metal, magnetism is suppressed and superconductivity appears. This general view is quite similar to the cuprate superconductor case. However, whether electrons or holes are actually doped [82-89], questions on how the Fermi surface evolves upon substitution, the reason for appearance of the superconducting phase, and why $\mathrm{T}_{\mathrm{c}}$ 's are very materials dependent (why they are so different) should be systematically studied.

In addition, other issues to be resolved are confirmation of pseudo-gaps [90-101], finding the relationship between pseudo-gaps and superconductivity, finding the origin of the $\mathrm{T}_{\mathrm{c}}$ enhancement in FeSe monolayer grown on $\mathrm{SrTiO}_{3}$ substrate [34, 107-111], confirmations of quantum critical points [101-106], material and doping dependence of orbital order, electronic structure studies of 1111 systems [35]. Information on these issues could provide clues to the microscopic mechanism. There are numerous issues to be resolved in the study of IBSCs. Finding additional IBSC materials and high quality single crystal growth of them is expected to play an essential role to the journey to the microscopic mechanism. ARPES will, as it has been, be one of the most important tools in the research.

\section{SUMMARY}

In this paper, we have briefly reviewed the research efforts by means of ARPES. The results so far in the first 
hand seem to suggest a close relationship between the nesting condition and the superconductivity, but it was pointed out that weak coupling approach alone cannot account for the superconductivity in IBSCs. At present, there is no theory that can account for the seemingly conflicting results and microscopic understanding of the superconductivity is not yet possible. To resolve such issue, more systematic studies focusing on deeper understanding of the system properties along with more accurate experimental results are necessary.

ARPES is expected to play an essential role in efforts to find the unified understanding of the superconducting mechanism. Advances in ARPES technology and high quality sample growth along with the existing information will allow us to shorten the time to obtain the microscopic mechanism.

\section{REFERENCES}

[1] P. J. Hirschfeld et al, "Gap symmetry and structure of Fe-based superconductors," Rep. Prog. Phys., vol. 74, pp. 124508, 2011.

[2] I. I. Mazin et al, "Unconventional Superconductivity with a sign Reversal in the Order Parameter of $\mathrm{LaFeAsO}_{1-\mathrm{x}} \mathrm{F}_{\mathrm{x}}$," Phys. Rev. Lett., vol. 101, pp. 057003, 2008.

[3] F. F. Tafti et al, "Sudden reversal in the pressure dependence of $T_{c}$ in the iron-based superconductor $\mathrm{KFe}_{2} \mathrm{As}_{2}$," Nat. Phys., vol. 9, pp. 349 , 2013.

[4] Ch. Platt et al, "Mechanism for a pairing state with time-reversal symmetry breaking in iron-based superconductors," Phys. Rev. B, vol. 85 , pp. 180502,2012

[5] S. Graser et al, "Near-degeneracy of several pairing channels in multiorbital models for the Fe pnictides," New J. Phys, vol. 11, pp. 025016, 2009

[6] R. Thomale et al, "Exotic d-Wave Superconducting state of Strongly Hole-Doped $\mathrm{K}_{\mathrm{x}} \mathrm{Ba}_{1-\mathrm{x}} \mathrm{Fe}_{2} \mathrm{As}_{2}$," Phys. Rev. Lett., vol. 107, pp. $117001,2011$.

[7] K. Suzuki et al, "Spin fluctuations and unconventional pairing in $\mathrm{KFe}_{2} \mathrm{As}_{2}$," Phys. Rev. B, vol. 84, pp. 144514, 2011

[8] S. Maiti et al, "Evolution of the superconducting state of Fe-based compounds with doping," Phys. Rev. Lett., vol. 107, pp. 147002, 2011.

[9] R. Fernades et al, "Suppression of superconductivity by Neel type magnetic fluctuations in the iron pnictides," Phys. Rev. Lett., vol. 110, pp. 117004, 2013.

[10] W. C. Lee et al, "Pairing state with a time-reversal symmetry breaking in FeAs-based superconductors," Phys. Rev. Lett., vol. 102, pp. 217002,2009

[11] V. Stanev et al, "Three-band superconductivity and the order parameter that breaks time-reversal symmetry," Phys. Rev. B, vol 81, pp. 134522, 2010.

[12] Ch. Platt et al, "Mechanism for a pairing state with time-reversal symmetry breaking in iron-based superconductors," Phys. Rev. B, vol. 85, pp. 180502, 2012

[13] P. Dai et al, "Magnetism and its microscopic origin in iron-based high temperature superconductors," Nat. Phys., vol. 8, pp. 709, 2012.

[14] S. Liang et al, "Nematic state of Pnictides Stabilized by interplay between Spin, Orbital and Lattice Degreeds of Freedom," Phys. Rev. Lett., vol. 111, pp. 047004, 2013.

[15] W. Lv et al, "Orbital ordering and unfrustrated $(\pi, 0)$ magnetism from degenerate double exchange in the iron pnictides," Phys. Rev. $B$, vol. 82, pp. 045125, 2010.

[16] W.-G. Yin et al, "Unified picture for magnetic correlations in iron-based superconductors," Phys. Rev. Lett., vol. 105, pp. 107004, 2010.

[17] R. Fernades et al, "Preemptive nematic order, pseudogap, and orbital order in the iron pnictides," Phys. Rev. B, vol. 85, pp. 024534,2012

[18] M. Yi et al, "Symmetry breaking orbital anisotropy observed for detwinned $\mathrm{Ba}\left(\mathrm{Fe}_{1-\mathrm{x}} \mathrm{Co}_{\mathrm{x}}\right)_{2} \mathrm{As}_{2}$ above the spin density wave transition," Proc. Natl. Acad. Sci., vol. 108, pp. 6878, 2011.
[19] M. Yi et al, "Electronic reconstruction through the structural and magnetic transitions in detwinned NaFeAs," New J. Phys., vol. 14 pp. 073019, 2012

[20] Y. Zhang et al, "Symmetry breaking via orbital-dependent reconstruction of electronic structure in detwinned NaFeAs," Phys. Rev. B, vol. 85, pp. 085121, 2012.

[21] J. Paglione et al, "High-temperature superconductivity in iron-based mateirals," Nat. Phys., vol. 6, pp. 1759, 2010.

[22] C.-H. Lee et al, "Effect of Structural Parameters on Superconductivity in Fluorine-Free $\mathrm{LnFeAsO}_{1-\mathrm{y}}(\mathrm{Ln}=\mathrm{La}, \mathrm{Nd}),{ }^{\prime} J$. Phys. Soc. Jpn., vol. 77, pp. 083704, 2008.

[23] Y Mizuguchi et al, "Anion height dependence of $\mathrm{T}_{\mathrm{c}}$ for the Fe-based superconductor," Superconductor Science and Technology, vol. 23, pp. 05401, 2010

[24] Kazuhiko Kuroki et al, "Pnictogen height as a possible switch between high- $T_{c}$ nodeless and low- $T_{c}$ nodal pairings in the iron-based superconductors," Phys. Rev. B, vol. 79, pp. 224511 , 2009.

[25] J. Zhao et al, "Structural and magnetic phase diagram of $\mathrm{CeFeAsO}_{1-\mathrm{x}} \mathrm{F}_{\mathrm{x}}$ and its relation to high-temperature superconductivity," Nat. Mater., vol. 7, pp. 953, 2008.

[26] http://www.physics.berkeley.edu/research/lanzara/research/pnictid e.html

[27] H. Kontani et al, "Origin of orthombic transition, magnetic transition, and shear-modulus softening in iron pnictide superconductors: Analysis based on the orbital fluctuations theory," Phys. Rev. B, vol. 84, pp. 024528, 2011.

[28] [28] H. Kontani et al, "Orbital fluctuation theory in iron-based superconductors: $\mathrm{s}^{++}$-wave superconductivity, structure transition, and impurity-induced nematic order," Sol. Stat. Comm., vol. 152, pp. 718, 2012.

[29] T. Saito et al, "Orbital fluctuation theory in iron pnictides: Effects of As-Fe-As bond angle, isotope substitution, and $\mathrm{Z}_{2}$ orbital pocket on superconductivity," Phys. Rev. B, vol. 82, pp. 144510, 2010.

[30] Y. Ono et al, "Structural transition, ferro-orbital order and its fluctuation-mediated S++-wave superconductivity in iron pnictides," Sol. Stat. Comm., vol. 152, pp. 701, 2012

[31] S. Kasahara et al, "Electronic nematicity above the structural and superconducting transition in $\mathrm{BaFe}_{2}\left(\mathrm{As}_{1-\mathrm{x}} \mathrm{P}_{\mathrm{x}}\right)_{2}$," Nature, vol. 486, pp 7403, 2012.

[32] M. Daghofer et al, "Orbital-weight redistribution triggered by spin order in the pnictides," Phys. Rev. B, vol. 81, pp. 180514, 2010.

[33] M. Yoshizawa et al, "Anomalous elastic behavior and its correation with superconductivity in iron-based superconductor $\mathrm{Ba}\left(\mathrm{Fe}_{1-\mathrm{x}} \mathrm{Co}_{\mathrm{x}}\right)_{2} \mathrm{As}_{2}$," Mod. Phys. Lett. B, vol. 26, pp. 1230011, 2012.

[34] D. Liu et al, "Electronic origin of high-temperature superconductivity in single-layer FeSe superconductor," Nat. Comm., vol. 3, pp. 1946, 2012.

[35] L. X. Yang et al, "Surface and bulk electronic structures of LaFeAsO studied by angle-resolved photoemission spectroscopy," Phys. Rev. B, vol. 82, pp. 104519, 2010.

[36] S. V. Borisenko et al, "Superconductivity without Nesting in LiFeAs," Phys. Rev. Lett., vol. 105, pp. 067002, 2010.

[37] A. Damascelli ,"Probing the Electronic Structure of complex systems by ARPES," Physica Scripta., T109, 2004.

[38] I. I. Mazin et al, "Superconductivity gets an iron boost," Nature, vol. 464 , pp. 183,2010

[39] M.M. Korshunov et al, "Theory of magnetic excitations in iron-based layered superconductors," Phys. Rev. B, vol. 78, pp. 1405092008

[40] A. V. Chubukov, "Pairing mechanism in Fe-based superconductors," Annu Rev. Condens. Mat. Phys., vol. 3, pp. 57, 2012.

[41] [41] F. Wang et al,"The electron-pairing mechanism of iron-based superconductors," Science, vol. 332, pp. 200, 2011.

[42] Q. Si et al, "Strong correlations and magnetic frustration in the high $\mathrm{T}_{\mathrm{c}}$ iron pnictides," Phys. Rev. Lett., vol. 101, pp. 076501, 2008.

[43] Q. Si et al,"Correlation effects in the iron pnictides," New J. Phys., vol. 11, pp. $045001,2009$.

[44] K. Seo et al, "Pairing symmetry in a two-orbital exchange coupling model of oxypnictides," Phys. Rev. Lett., vol. 101, pp. 206404 2008.

[45] F. Ma et al,"Arsenic-bridged antiferromagnetic superexchange interactions in LaFeAsO," Phys. Rev. B, vol. 78, pp. 224517, 2008

[46] M. M. Qazilbash et al, "Electronic correlations in the iron pnictides," Nat. Phys., vol. 5, pp. 647, 2009.

[47] T. Terashima et al., "Fermi surface and mass enhancement in $\mathrm{KFe}_{2} \mathrm{As}_{2}$ from de Haas-Van Alphen effect measurements," J. Phys. Soc. Jpn., vol. 79, pp. 053702, 2010 
[48] K. Nakamura et al, "Ab initio derivation of low-energy model for iron-based superconductors LaFeAsO and LaFePO,"J. Phys. Soc. Jpn., vol. 77, pp. 093711, 2008.

[49] D. S. Inosov et al, "Crossover from weak to strong pairing in unconventional superconductors," Phys. Rev. B, vol. 83, pp. 213520 2011.

[50] Z. P. Yin et al, "Kinetic frustration and the nature of the magnetic and paramagnetic states in iron pnictides and iron chalcogenides,' Nat. Mater., vol. 10, pp. 932, 2011.

[51] C. He et al, "Electronic-structure-driven magnetic and structure transitions in superconducting NaFeAs single crystals measured by angle-resolved photoemission spectroscopy," Phys. Rev. Lett., vol. 105 , pp. 117002, 2010.

[52] A. Nicholson et al., "Role of degeneracy, hybridization, and nesting in the properties of multi-orbital systems," Phys. Rev. B, vol. 84, pp 094519,2011

[53] M. Y. Wang et al, "Spin waves and magnetic exchange interactions in insulating $\mathrm{Rb}_{0.89} \mathrm{Fe}_{1.58} \mathrm{Se}_{2}$," Nat. Comm., vol. 2, pp. 580, 2011.

[54] M. S. Liu et al, "Nature of magnetic excitations in superconducting $\mathrm{BaFe}_{1.9} \mathrm{Ni}_{0.1} \mathrm{As}_{2}$." Nat. Phys., vol. 8, pp. 376, 2012.

[55] H. Park et al., "Magnetic excitation spectra in $\mathrm{BaFe}_{2} \mathrm{As}_{2}$ : A two-particle approach within a combination of the density functional theory and the dynamical mean-field theory method," Phys. Rev. Lett., vol. 107, pp. 137007, 2011.

[56] H. Gretarsson et al, "Revealing the dual nature of magnetism in iron pnictides and iron chalcogenides using $\mathrm{x}$-ray emission spectroscopy," Phys. Rev. B, vol. 84, pp. 100509, 2011.

[57] F. Bondino et al, "Evidence for Strong Itinerant Spin Fluctuations in the Normal State of $\mathrm{CeFeAsO}_{0.89} \mathrm{~F}_{0.11}$ Iron-Oxypnictide Superconductors," Phys. Rev. Lett., vol. 101, pp. 267001, 2008.

[58] M. Yi et al, "Electronic structure of the $\mathrm{BaFe}_{2} \mathrm{As}_{2}$ family of iron-pnictide superconductors," Phys. Rev. B, vol. 80, pp. 024515 , 2009

[59] W. Malaeb et al, "Abrupt change in the energy gap of superconducting $\mathrm{Ba}_{1-\mathrm{x}} \mathrm{K}_{\mathrm{x}} \mathrm{Fe}_{2} \mathrm{As}_{2}$ single crystals with hole doping," Phys. Rev. B, vol. 86, pp. 165117, 2012.

[60] N. Xu et al, "Effects of Ru substitution on electron correlations and Fermi-surface dimensionality," Phys. Rev. B, vol. 86, pp. 064505 , 2012 .

[61] [61] Z. R. Ye et al, "Orbital selective correlations between nesting/scattering/Lifshitz transition and the superconductivity in $\mathrm{AFe}_{1-\mathrm{x}} \mathrm{Co}_{\mathrm{x}} \mathrm{As}(\mathrm{A}=\mathrm{Li}, \mathrm{Na}), "$ arXiv 1303.0682v1.

[62] [62] S. V. Borisenko et al, "Superconductivity without nesting in LiFeAs,” Phys. Rev. Lett., vol. 105, pp. 067002, 2010.

[63] [63] T. Qian et al, "Absence of holelike Fermi surface in superconducting $\mathrm{K}_{0.8} \mathrm{Fe}_{1.7} \mathrm{Se}_{2}$ revealed by ARPES," Phys. Rev. Lett., vol. 106, pp. 187001, 2011.

[64] [64] Y. Zhang et al, "Nodeless superconducting gap in $\mathrm{A}_{\mathrm{x}} \mathrm{Fe}_{2} \mathrm{Se}_{2}(\mathrm{~A}=\mathrm{K}, \mathrm{Cs})$ revealed angle-resolved photoemission spectroscopy," Nat. Mater., vol. 10, pp. 2981, 2011.

[65] Y. Zhang et al, "Heavily electron-doped electronic structure and isotropic superconducting gap in $\mathrm{A}_{\mathrm{x}} \mathrm{Fe}_{2} \mathrm{Se}_{2}(\mathrm{~A}=\mathrm{K}, \mathrm{Cs})$," Nat. Mater. vol. 10, pp. 273, 2011

[66] D. Mou et al, "Distinct Fermi surface topology and nodelss superconducting gap in a $\left(\mathrm{Tl}_{0.58} \mathrm{Rb}_{0.42}\right) \mathrm{Fe}_{1.72} \mathrm{Se}_{2}$ superconductor," Phys. Rev. Lett., vol. 106, pp. 107001, 2011.

[67] H. Miao et al, "Coexistence of orbital degeneracy lifting and superconductivity in iron-based superconductors," Phys. Rev. B, vol. 89, pp. 220503, 2014.

[68] S. V. Borisenko et al, "One-Sign Order Parameter in Iron Based Superconductor," Symmetry, vol. 4, pp. 251, 2012.

[69] H. Miao et al, "Isotropic superconducting gaps with enhanced pairing on electron Fermi surfaces in $\mathrm{FeTe}_{0.55} \mathrm{Se}_{0.45}$," Phys. Rev. B, vol. 85, pp. 094506, 2012.

[70] N. Xu et al, "Angle-resolved photoemission observation of isotropic superconducting gaps in isovalent Ru-substituted $\mathrm{Ba}\left(\mathrm{Fe}_{0.75} \mathrm{Ru}_{0.25}\right)_{2} \mathrm{As}_{2}$," Phys. Rev. B, vol. 87, pp. 094513, 2013.

[71] H. Ding et al, "Observation of Fermi-surface-dependent nodeless superconducting gaps in $\mathrm{Ba}_{0.6} \mathrm{~K}_{0.4} \mathrm{Fe}_{2} \mathrm{As}_{2}$," Eur. Phys. Lett., vol. 83, pp. 47001, 2008.

[72] K. Terashima et al, "Fermi surface nesting induced strong pairing in iron-based superconductors," Proc. Natl. Acad. Sci., vol. 106, pp 7330, 2009.

[73] Y. B. Huang et al, "Angle-resolved photoemission studies of the superconducting gap symmetry in Fe-based superconductors," Am. Ist. Phys. Adv., vol. 2, pp. 041409, 2012.
[74] T. Shimojima et al,"Orbital Independent Superconducting Gaps in Iron Pnictides," Science, vol. 332, pp.564, 2011.

[75] K. Umezawa et al, "Unconventional Anisotropic s-Wave Superconducting Gaps of the LiFeAs Iron-Pnictide Superconductor,' Phys. Rev. Lett., vol. 108, pp. 037002, 2012.

[76] T. Kondo et al, "Momentum Dependence of the Superconducting Gap in NdFeAsO0.9F0.1 Single Crystals Measured by Angle Resolved Photoemission Spectroscopy," Phys. Rev. Lett., vol. 101, pp. 147003,2008

[77] K. Okazaki et al, "Octet-Line Node Structure of Superconducting Order Parameter in $\mathrm{KFe}_{2} \mathrm{As}_{2}$," Science, vol. 337, pp. 1314, 2012.

[78] Y. Zhang et al, "Nodal superconducting-gap structure in ferropnictide superconductor $\mathrm{BaFe}_{2}\left(\mathrm{As}_{0.7} \mathrm{P}_{0.3}\right)_{2}$," Nat. Phys., vol. 8 , pp. 2248, 2012.

[79] S. O. Diallo et al, "Itinerant magnetic excitations in antiferromagnetic CaFe2As2," Phys. Rev. Lett., vol. 102, pp. 187206, 2009.

[80] R. A. Ewings et al, "Itinerant spin excitations in $\mathrm{SrFe}_{2} \mathrm{As}_{2}$ measured by inelastic neutron scattering," Phys. Rev. B, vol. 83, pp. 214519 , 2011.

[81] J. Zho et al. "Spin waves and magnetic exchange interaction in $\mathrm{CaFe}_{2} \mathrm{As}_{2}$," Nat. Phys., vol. 5, pp. 555, 2011.

[82] L. W Harriger et al, "Nematic spin fluid in the tetragonal phase of $\mathrm{BaFe}_{2} \mathrm{As}_{2}$," Phys. Rev. B, vol. 84, pp. 054544, 2011

[83] H. Chen et al, "Coexistence of the spin-density wave and superconductivity in $\mathrm{Ba}_{1-\mathrm{x}} \mathrm{K}_{\mathrm{x}} \mathrm{Fe}_{2} \mathrm{As}_{2}$," Euro. Phys. Lett., vol. 85, pp. 17006, 2009.

[84] D. K. Pratt et al, "Coexistence of competing Antiferromagnetic and Superconducting Phases in the Underdoped $\mathrm{Ba}\left(\mathrm{Fe}_{0.953} \mathrm{Co}_{0.047}\right)_{2} \mathrm{As}_{2}$ Compound Using X-ray and Neutron Scattering Techniques," Phys. Rev. Lett., vol. 103, pp. 087001, 2009.

[85] S. Kasahara et al, "Evolution from non-Fermi to Fermi-liquid transport via isovalent doping in $\mathrm{BaFe}_{2}\left(\mathrm{As}_{1-\mathrm{x}} \mathrm{P}_{\mathrm{x}}\right)_{2}$ superconductors," Phys. Rev. B, vol. 82, pp. 184519, 2010.

[86] Z. R. Ye et al, "Doping dependence of the electronic structure in phosphorus-doped ferropnictide superconductor $\mathrm{BaFe}_{2}\left(\mathrm{As}_{1-\mathrm{x}} \mathrm{P}_{\mathrm{x}}\right)_{2}$ studied by angle-resolved photoemission spectroscopy," Phys. Rev. $B$, vol. 86, pp. 035136, 2012.

[87] M. J. Eom et al, "Evolution of transport properties of $\mathrm{BaFe}_{2-\mathrm{x}} \mathrm{Ru}_{\mathrm{x}} \mathrm{As}_{2}$ in a wide range of isovalent Ru substitution," Phys. Rev. B, vol. 85 , pp. 024536,2012

[88] K. Kirshenbaum et al, "Universal pair-breaking in transition-metal-substituted iron-pnictide superconductors," Phys. Rev. B, vol. 86, pp. 14505, 2012.

[89] A. S. Sefat et al, "Superconductivity at $22 \mathrm{~K}$ in Co-Doped $\mathrm{BaFe}_{2} \mathrm{As}_{2}$ Crystals," Phys. Rev. Lett., vol. 101, pp. 117004, 2008.

[90] H.-Y. Liu et al, "Pseudogap and Superconducting Gap in $\operatorname{SmFeAs}\left(\mathrm{O}_{1-\mathrm{x}} \mathrm{F}_{\mathrm{x}}\right) \quad$ Superconductor from Photoemission Spectroscopy," Chin. Phys. Lett., vol. 25, pp. 3761, 2008.

[91] T. Sato et al, "Superconducting Gap and Pseudogap in Iron-Based Layered Superconductor $\mathrm{La}\left(\mathrm{O}_{1-\mathrm{x}} \mathrm{Fx}\right) \mathrm{FeAs}$," J. Phys. Soc. Jpn., vol. 77, pp. 063708, 2008.

[92] H. Ikeda et al., "Pseudogap and Superconductivity in Iron-Based Layered Superconductor Studied by Flucutation-Exchange Approximation," J. Phys. Soc. Jpn., vol. 77, pp. 123707, 2008.

[93] T. Shimojima et al, "Pseudogap formation above the superconducting dome in iron pnictides," Phys. Rev. B, vol. 89, pp. 045101, 2014

[94] Y. Ishida et al, "Unusual Pseudogap Features Observed in Iron Oxypnictide Superconductors," J. Phys. Soc. Jpn., vol. 77, pp. 61, 2008.

[95] K. Ahilan et al, " $\mathrm{F}^{19}$ NMR investigation of the iron pnictide superconductor $\mathrm{LaFeAsO}_{0.89} \mathrm{~F}_{0.11}$," Phys. Rev. B, vol. 78, pp. 100501, 2008.

[96] D. R. Garcia et al, "Core-level and valence-band study using angle-integrated photoemission on $\mathrm{LaFeAsO}_{0.9} \mathrm{~F}_{0.1}$," Phys. Rev. B, vol. 78, pp. 245119, 2008

[97] T. Mertelj et al, "Distinct Pseudogap and Quasiparticle Relaxation Dynamics in the Superconducting State of Nearly Optimally Doped $\mathrm{SmFeAsO}_{0.8} \mathrm{~F}_{0.2}$ Single Crystals," Phys. Rev. Lett., vol. 102, pp. 117002,2009

[98] M. A. Tanatar et al, "Pseudogap and its critical point in the heavily doped $\mathrm{Ba}\left(\mathrm{Fe}_{1-\mathrm{x}} \mathrm{Co}_{\mathrm{x}}\right)_{2} \mathrm{As}_{2}$ from c-axis resistivity measurements," Phys. Rev. B, vol. 82, pp. 134528, 2010.

[99] S. H. Baek et al, "Pseudogap-like phase in $\mathrm{Ca}\left(\mathrm{Fe}_{1-\mathrm{x}} \mathrm{Co}_{\mathrm{x}}\right)_{2} \mathrm{As}_{2}$ revealed by 75As NQR," Phys. Rev. B, vol. 84, pp. 094510, 2011. 
[100]Y. C. Wen et al, "Gap Opening and Orbital Modification of Superconducting FeSe above the Structural Distortion," Phys. Rev. Lett., vol. 108, pp. 267002, 2012.

[101]S. J. Moon et al, "Infrared Measurement of the Pseudogap of P-Doped and Co-doped High-Temperature $\mathrm{BaFe}_{2} \mathrm{As}_{2}$ Superconductors," Phys. Rev. Lett., vol. 109, pp. 027006, 2012.

[102]K. Hashimoto et al, "A sharp peak of the zero-temperature penetration depth at optimal composition in $\mathrm{BaFe}_{2}\left(\mathrm{As}_{1-\mathrm{x}} \mathrm{P}_{\mathrm{x}}\right)_{2}$," Science, vol. 336, pp. 1554, 2012.

[103]P. Walmsley et al, "Quasiparticle mass enhancement close to the quantum critical point in $\mathrm{BaFe}_{2}\left(\mathrm{As}_{1-\mathrm{x}} \mathrm{P}_{\mathrm{x}}\right)_{2}$," arXiv:1303.3396

[104]J. K. Dong et al, "Quantum criticality and nodal superconductivity in the FeAs-based superconductor $\mathrm{KFe}_{2} \mathrm{As}_{2}$," Phys. Rev. Lett., vol. 104, pp. 087005, 2010.

[105]J. Dai et al, "Iron pnictides as a new setting for quantum criticality," Proc. Natl. Acad. Sci., vol. 106, pp. 4118, 2009.

[106]Y. Nakai et al, "Unconventional superconductivity and antiferro magnetic quantum critical behavior in the isovalent doped $\mathrm{BaFe}_{2}\left(\mathrm{As}_{1-\mathrm{x}} \mathrm{P}_{\mathrm{x}}\right)_{2}$," Phys. Rev. Lett., vol. 105, pp. 107003, 2010.
[107]F. Zheng et al, "Antiferromagnetic FeSe monolayer on $\mathrm{SrTiO}_{3}$ : The charge doping and electric field effects," Scientific Reports, vol. 3, pp. 2213, 2013.

[108]Q.-Y. Wang et al, "Interface-induced High-Temperature Superconductivity in Single Unit-Cell FeSe Films on $\mathrm{SrTiO}_{3}$, , Chin Phys. Lett., vol. 29, pp. 037402, 2012.

[109]S.-L He et al, "Phase diagram and high temperature superconductivity at $65 \mathrm{~K}$ in tuning carrier concentration of single-layer FeSe films," Nat. Mater., vol. 12, pp. 605, 2013.

[110]S. Y. Tan. et al, "Interface-induced superconductivity and strain-dependent spin density wave in $\mathrm{FeSe} / \mathrm{SrTiO}_{3}$ thin films," Nat. Mater., vol. 12, pp. 634, 2013.

[111]Y. Y. Xiang et al, "High-temperature superconductivity at the FeSe/SrTiO 3 interface," Phys. Rev. B, vol. 86, pp. 134508, 2012. 